\title{
Experimental Evidence That Hubble's Parameter Could Be Reflected in Local Physical and Chemical Reactions: Support for Mach's Principle of Imminence of the Universe
}

\author{
Michael A. Persinger \\ Laurentian University, Sudbury P3E 2C6, Ontario, Canada \\ E-mail address: mpersinger@laurentian.ca
}

\begin{abstract}
Mach's principle of immanence of the universe requires the behavior of local matter to depend upon the remainder of the universe. Hubble's constant could be employed to calculate the inference of this condition. More than a decade ago specific types of temporally patterned magnetic fields generated by serial $3 \mathrm{~ms}$ point durations were shown to produce conspicuous biochemical effects. When the product of this duration and the diameter of a proton was divided into Planck's Length the estimated Hubble' parameter was $66.7 \pm 1.3 \mathrm{~km} \cdot \mathrm{s}^{-1} \cdot$ MParsec $^{-1}$ which is remarkably similar to the current value of $67.8 \mathrm{~km} \cdot \mathrm{s}^{-1}$. Photon emissions from chemoluminescent reactions and discrete shifts in $\mathrm{pH}$ within spring water during exposure to specific patterns of magnetic fields revealed optimal responses with remarkably similar z-transformations when point durations of the constructive voltages were $3 \mathrm{~ms}$ or $1 \mathrm{~ms}$ but not 2 , 4, or $5 \mathrm{~ms}$. Cumulative results indicate proton $(3 \mathrm{~ms})$ and electron $(\sim 1.5$ ms) processes. These results support Mach's contention and indicate that relatively accurate inferences of the Hubble parameter might be obtained with more precise instrumentation on the terrestrial surface.
\end{abstract}

Keywords: Hubble' constant; Planck's length expansions; protons and electrons; point durations; Mach's principle; local estimates

\section{INTRODUCTION}

During the late $19^{\text {th }}$ century Ernst Mach [1] proposed the principle of immanence of the universe whereby properties of local matter depend on the presence of the remainder of the universe. In addition, the natural laws governing the largest scale of space-time should be intrinsically related to the operations and patterns within the smallest spatial-temporal scale. Estimates of the expansion of the largest space-time scale, as reflected in Hubble's constant, has ranged between 50 and $100 \mathrm{~km} \cdot \mathrm{s}^{-1} \cdot \mathrm{MParsec}^{-1}$. If Mach's principle is applicable then the expansion of local space should be commensurate with and predict the quantitative value of Hubble's constant with precision approaching that from more stellar measurements. In this paper evidence for this possibility is presented and a potential procedure for its demonstration is explained.

According to the theory developed by Persinger and Koren [2] the time for the expansion of any space and the matter occupied within it should occur as increments of 
Planck's length which is $\sqrt{ }\left(\hbar \mathrm{Gc}^{-3}\right)$ where $\hbar$ is the modified Planck's constant, $\mathrm{G}$ is the gravitational constant and $\mathrm{c}$ is the velocity of light in a vacuum. The duration of expansion would not be continuous but reflect discrete increments. Assuming a median value of $75(\mathrm{~km}$. $\left.\mathrm{s}^{-1}\right) \cdot \mathrm{MParsec}^{-1}$ and $3.09 \cdot 10^{22} \mathrm{~m}$ per Parsec, the Hubble expansion parameter $\mathrm{H}$ is about $2.4 \cdot 10^{-}$ ${ }^{18} \mathrm{~s}^{-1}$. The assumed range has been between 1.6 and $3.2 \cdot 10^{-18} \mathrm{~s}^{-1}$. Multiplication by any spatial extent (length) produces a velocity that when divided into Planck's length results in a specific duration.

When this relationship is applied to the boundaries of space a striking condition emerges. The theoretical time required for a length equivalent to the total set, the universe $\left(\sim 1.25 \cdot 10^{26} \mathrm{~m}\right)$, to expand one Planck's length is the product of this value and $2.4 \cdot 10^{-18} \mathrm{~s}^{-1}$ divided into Planck's length $\left(1.62 \cdot 10^{-35} \mathrm{~m}\right)$ and approaches Planck's time $\left(5.4 \cdot 10^{-44} \mathrm{~s}\right)$. The time required for a length approaching Planck's length to expand one Planck's length would approach $4.1 \cdot 10^{17} \mathrm{~s}$ or 13 billion years, the approximate age of the universe. In other words the largest length expands "instantaneously" while the shortest length requires the age of the universe to be manifested once.

Although one cannot easily test this boundary condition at each of the extremes application to more tractable fundamental lengths, such as the proton or electron, might be both revealing and capable of at least indirect testing. Here the length of a proton is assumed to be $2.5 \cdot 10^{-15} \mathrm{~m}$ (approximately twice the Compton wavelength). For a proton the velocity of expansion, based upon the intermediate value of Hubble's parameter, would be $6 \cdot 10^{-33} \mathrm{~m} \cdot \mathrm{s}^{-1}$. The time to expand one Planck's length would be $1.62 \cdot 10^{-35} \mathrm{~m}$ divided by $6 \cdot 10^{-33} \mathrm{~m} \cdot \mathrm{s}^{-1}$ or $\sim 2.7$ ms. If the values for $\mathrm{v}_{\min }\left(50 \mathrm{~km} \cdot \mathrm{s}^{-1} \cdot \mathrm{MParsec}{ }^{-1}\right)$ and $\mathrm{v}_{\max }\left(100 \mathrm{~km} \cdot \mathrm{s}^{-1} \cdot \mathrm{MParsec}^{-1}\right)$ are employed the value ranges from $\sim 2$ to $4 \mathrm{~ms}$. For the electron with a length of $4.86 \cdot 10^{-15} \mathrm{~m}$ (twice the classical radius) the product with $2.4 \cdot 10^{-18} \mathrm{~s}^{-1}$ is $11.66 \cdot 10^{-33} \mathrm{~m} \cdot \mathrm{s}^{-1}$ and when divided into Planck's length results in the durations in a range from $\sim 1.0$ to $1.8 \mathrm{~ms}$.

For more than a decade we have shown that cell cultures, planarian, and vertebrates respond maximally to weak physiologically-patterned magnetic fields in the range of $\mu \mathrm{T}$ when the point duration that composed the patterns generated by computer software and transformed from digital-to-analogue signals was $3 \mathrm{~ms}$. The same fields but generated at different point durations, $2 \mathrm{~ms}$ or $4 \mathrm{~ms}$ to $10 \mathrm{~ms}$ were not effective [3-6]. Unlike traditional methods of creating magnetic fields from function generators we developed a computer software that transformed columns of numbers between 1 and 256 through custom constructed digital to analogue converters to values between $-5 \mathrm{~V}$ and $+5 \mathrm{~V}$, where the value $127=0 \mathrm{~V}$. With the $\mathrm{Y}$ axis ranging from 0 to 256 and the $\mathrm{x}$ axis representing the duration each number or voltage was created by the computer one could generate a string of voltages and hence any pattern imagined by theoretical or empirical reasoning. The point duration is the time a given integer value between 0 and 256 and hence the resulting voltage is presented to digital-to-analogue converter and subsequently to the solenoids that generate the magnetic fields.

The two temporal patterns of magnetic fields employed were selected because of their powerful effects upon living systems for producing analgesia and influencing cell division and viability [3-6]. The first pattern was composed of 849 points and exhibits a decelerating complex frequency modulation. The second pattern is composed of 230 points and exhibits an accelerating complex frequency modulation. They are both depicted in Figure 1. 
AD Configuration

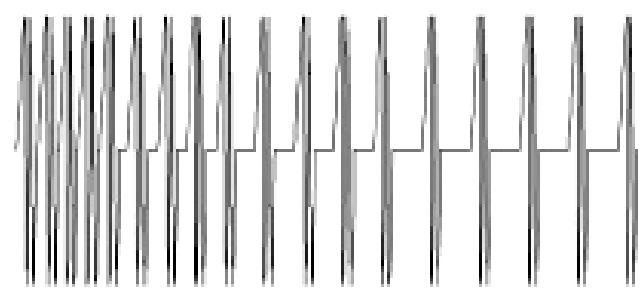

(a)
DI Configuration

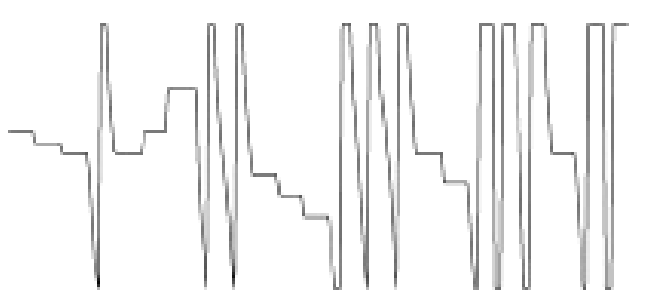

(b)

Figure 1. (a) The decelerating frequency-modulated pattern and (b) the accelerating frequencymodulated patterns. Both have been employed in previous experiments to demonstrate the efficacy of $3 \mathrm{~ms}$ point durations (adapted from [7]).

Assuming the $3 \mathrm{~ms}$ duration is discrete $(3.0 \mathrm{~ms})$ and reflects an integer rather than the limitation of the software to employ only whole units, the application of the equation predicts that Hubble's constant would be $66.7 \mathrm{~km} \cdot \mathrm{s}^{-1} \cdot \mathrm{MParsec}^{-1}$. Considering the estimated range in the width of a proton, 1 standard deviation from that mean would be $\sim 1.3 \mathrm{~km} \cdot \mathrm{s}^{-1} \cdot \mathrm{MParsec}^{-1}$. Consequently the range in the predicted value from surface terrestrial phenomena is within measurement error of the 2012 Planck Mission's value of $67.8 \pm 0.77 \mathrm{~km} \cdot \mathrm{s}^{-1} \cdot \mathrm{MParsec}^{-1}$. The latter value predicts that the duration required for an electron to expand one Planck's length should be $1.5 \mathrm{~ms}$. If this were a fundamental effect then the $\sim 3.0 \mathrm{~ms}$ and $\sim 1.5 \mathrm{~ms}$ point durations should be reflected within all physical chemical systems when the appropriately patterned magnetic field is applied. Here I review this effect from previous research involving photon generation and the current experiment involving shifts in $\mathrm{pH}$ within aqueous systems when magnetic fields with different point durations were applied.

\section{PREVIOUS EXPERIMENTAL DEMONSTRATIONS OF THE IMPORTANCE OF FIELD POINT DURATIONS}

While investigating methods for producing macroscopic entanglement between fields of photons, Dotta and Persinger [7] found that simultaneous exposure of two photochemical reactions within circular arrays of 8 solenoids that generated magnetic fields with specific rates of change in angular velocities produced a doubling of the yield of photons as measured by photomultiplier tubes. The two loci were separated by $10 \mathrm{~m}$ and in different rooms. The effect was evident up to $3 \mathrm{~km}$ (maximum distance tested to date). The photon emissions were produced by injecting $0.1 \mathrm{cc}$ of sodium hypochlorite into $6 \mathrm{cc}$ of hydrogen peroxide. When this amount was injected into the hydrogen peroxide simultaneously in the two locations the yield was the same as if $0.2 \mathrm{cc}$ of sodium hypochlorite was injected into either location only. There was a doubling of the photon output that was quantitatively predictable and visually conspicuous.

The most striking effect, in addition to the transience of about $8 \mathrm{~min}$ for the "entanglement" or excessive correlation between the loci was the observation that the effect was only apparent when the point durations of the optimal field sequence was either $1 \mathrm{~ms}$ or 3 ms. A representation of the results reported by Koren et al [8] is shown in Figure 2 for integers of $1 \mathrm{~ms}$ through $5 \mathrm{~ms}$ point durations. The value for $5 \mathrm{~ms}$ was effectively 0 . The 
doubling of photon emissions was only evident when the point durations of the optimal magnetic fields required to produce the effect were approximate to the theoretical duration for an electron $(\sim 1 \mathrm{~ms})$ and proton $(\sim 3 \mathrm{~ms})$ to expand one Planck's length. Based upon multiple experiments the patterned magnetic fields created from $3 \mathrm{~ms}$ point durations affect protonrelated biochemical, cellular, and analgesic responses in organisms. On the other hand the same field generated by $1 \mathrm{~ms}$ point durations appear to influence electron-related processes.

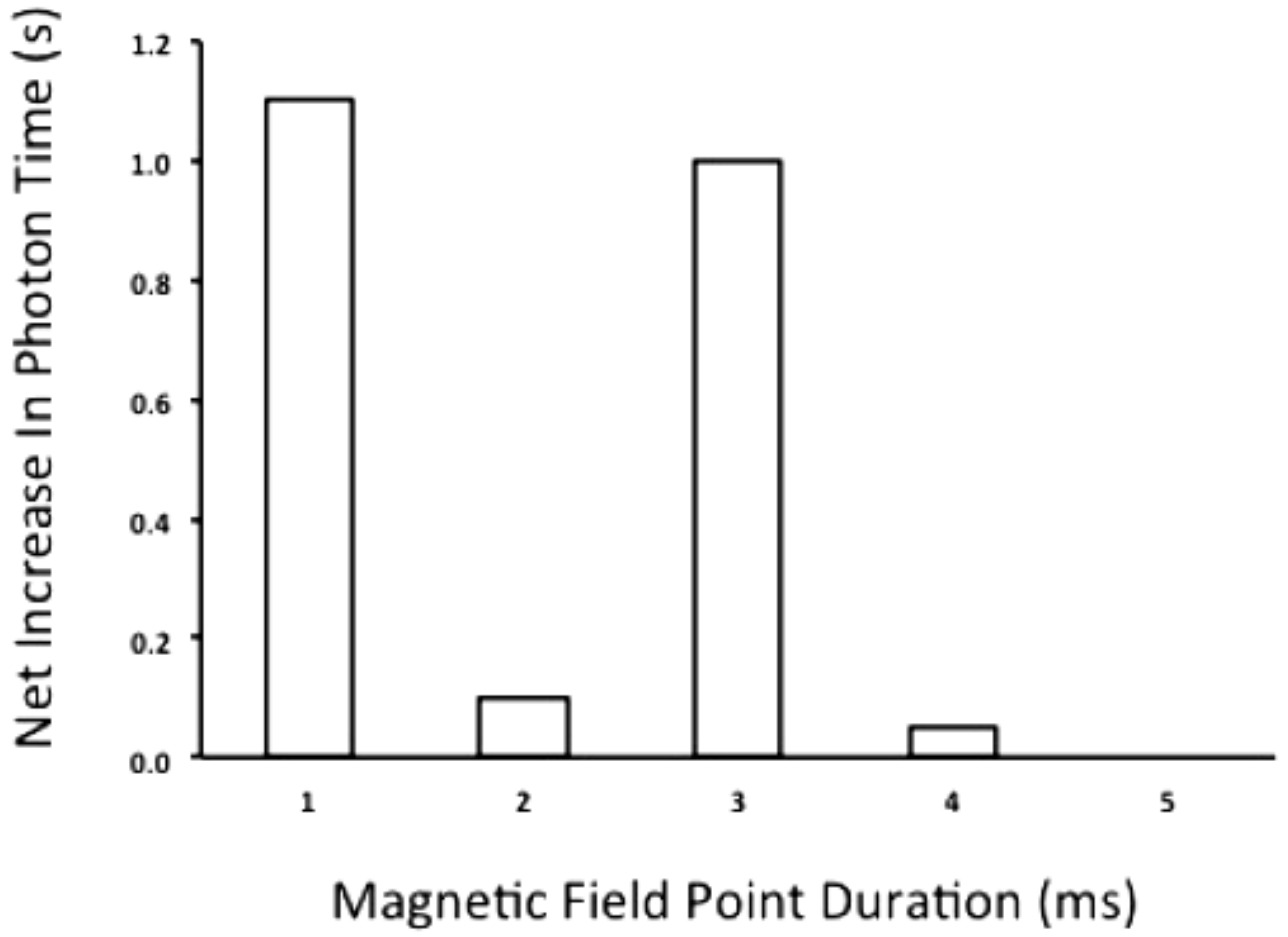

Figure 2. Net increase in photon emission durations when two loci shared the same configuration magnetic fields with different point durations (horizontal axis, in ms) and received the chemical reactant to emit the photons simultaneously.

\section{THE PRESENT STUDY: SHIFTS IN H $^{+}$IN SPRING WATER BY 1 MS AND 3 MS POINT DURATION MAGNETIC FIELDS}

To discern if the 1, $3 \mathrm{~ms}$ duration effect was present during the normal trend towards mild alkalization of $\mathrm{pH}$ in spring water [9] when poured from containers into open beakers, $100 \mathrm{cc}$ of spring water in beakers were exposed to a frequency modulated magnetic field pattern for $12 \mathrm{~h}$. The $\mathrm{pH}$ values for the water in each experiment were recorded separately once per minute (720 measurements) by Dr. Daq systems (Pico Technology, United Kingdom) which are sensitive to the $.01 \mathrm{pH}$ unit. The data were recorded continuously on a laptop operating the appropriate software.

The point durations for the same pattern was either $1,2,3,4$, or $5 \mathrm{~ms}$. The strength of the field generated through the $100 \mathrm{cc}$ of water was about 5 to $10 \mu \mathrm{T}$. The field was generated by four pairs of reed relays that operated as solenoids. The containers within which the solenoids were fixed were separated by the width of the beaker. A more detailed description 
of the exposure equipment can be found elsewhere [9]. All exposures were completed in triplicate. The means of the values per condition were employed as the data.

The results are shown in Figure 3. There were no significant differences in $\mathrm{pH}$ (7.82 to 7.83 for all point durations) during the first 8 minutes of exposures for the different point durations. However by the end of the $12 \mathrm{hr}$ of exposure the water that had been exposed to the 1 and $3 \mathrm{~ms}$ point durations displayed less alkalinity than the other point durations. The primary inflection point occurred around $3 \mathrm{hrs}$.

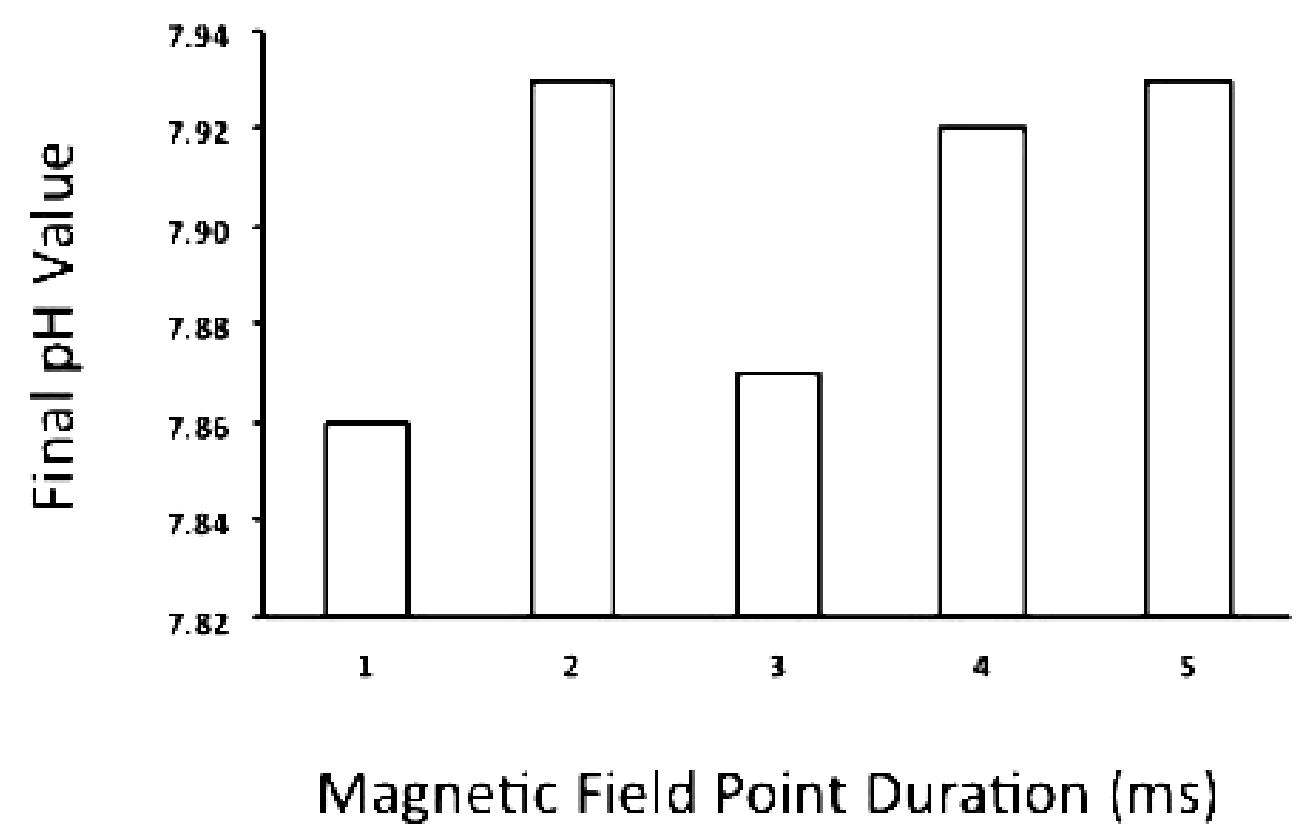

Figure 3. Final mean $\mathrm{pH}$ values for $100 \mathrm{cc}$ of spring water after exposure for 12 hour to frequencymodulated magnetic field (Figure 1a) generated by different point durations (in $\mathrm{ms}$ ).

According to the traditional equation $\mathrm{M}=10^{-\mathrm{pH}}$, the net difference between the $\mathrm{pH}$ values for the water exposed to the 1 and $3 \mathrm{~ms}$ point durations and the remaining point durations was $2 \cdot 10^{-9} \mathrm{M}$. With $6.023 \cdot 10^{23}$ molecules per Mole and 5.55 Moles of water in 100 cc, this is equivalent to $8.3 \cdot 10^{15}$ protons. Considering the effect was primary during the first 3 hr $\left(1.08 \cdot 10^{4} \mathrm{~s}\right)$, this would be equivalent $7.69 \cdot 10^{11}$ protons per $\mathrm{s}$. This means that one proton was affected once every $1.3 \cdot 10^{-12} \mathrm{~s}$ or a picosecond. This duration is remarkably similar to the multiple experimental measurements of the duration of $\mathrm{H}_{3} \mathrm{O}^{+}$(hydronium) ion before the $\mathrm{H}^{+}$ moves to another water molecule [10].

\section{DISCUSSION}

The results of two separate experiments employing different physical systems, photon emission during aqueous chemiluminescent reactions and natural shifts in $\mathrm{pH}$ of spring water during exposure to open air, both revealed the particular enhancement of their respective effects when the point durations that generated the magnetic fields were $3 \mathrm{~ms}$ or $1 \mathrm{~ms}$. The effects are not likely to be artifacts of exposure equipment. The photon emission study involved a circular array of 8 solenoids generating moving focused magnetic fields around the 
perimeter with varying angular accelerations while the $\mathrm{pH}$ shift study occurred within the same magnetic field pattern generated between clusters of four pairs of opposing solenoids.

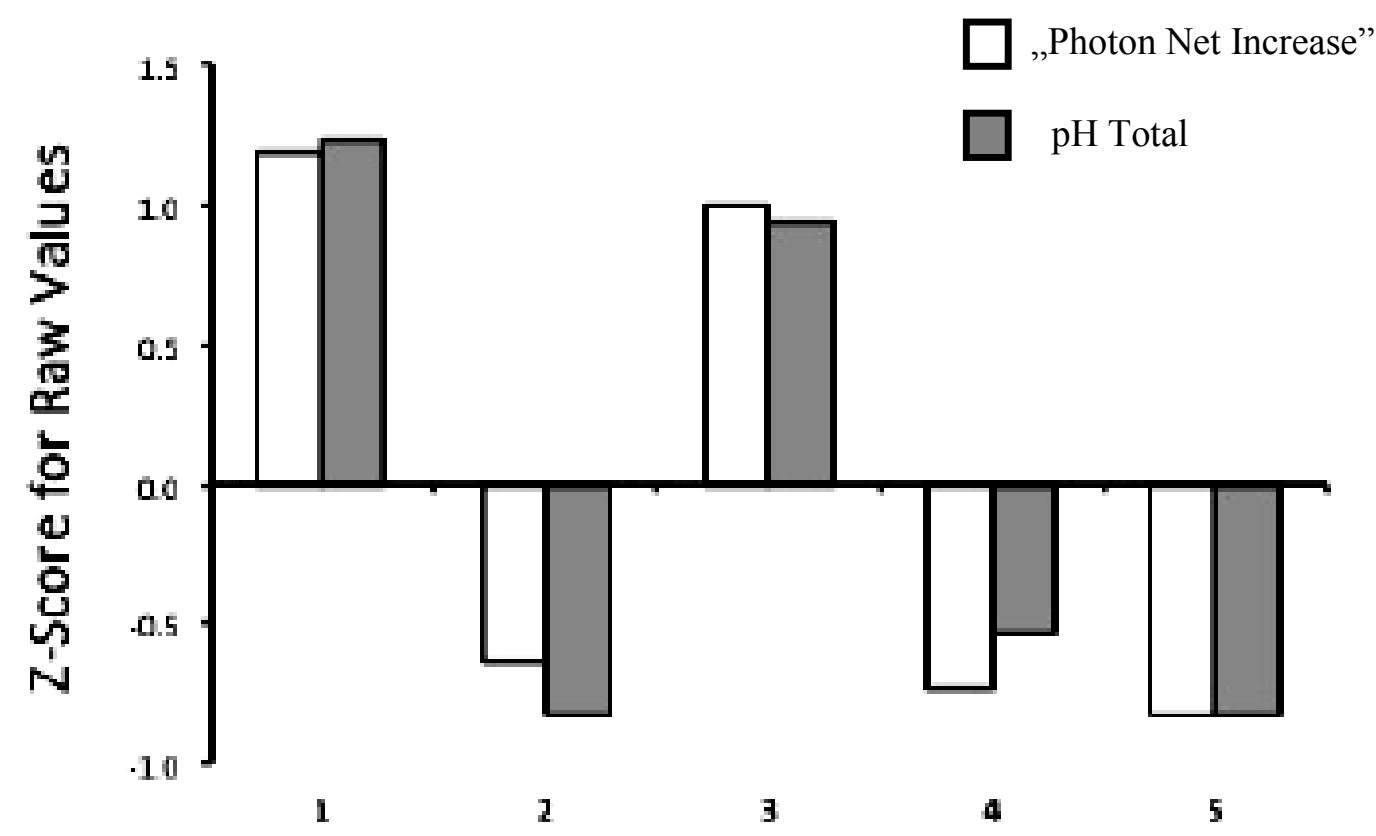

Magnetic Field Point Duration

Figure 4. Z-scores of raw values for the change in durations of photon emissions and for the alterations in $\mathrm{pH}$ in spring water as a function of the point durations (in ms) that composed the frequency-modulated magnetic fields to which the samples were exposed.

In order to compare the standardized effect from both experiments because one involved increased duration of photon emission and the other involved shifts in $\mathrm{pH}$ the raw values within an experiment were transformed to z-scores. Because the $\mathrm{pH}$ experiment involved less quantitative value rather than increased quantitative value, the former was multiplied by -1 . The results are shown in Figure 4. For both experiments the relative efficacies of the $1 \mathrm{~ms}$ and $3 \mathrm{~ms}$ point durations compared to 2 , 4 , or $5 \mathrm{~ms}$ point durations were remarkably similar.

\section{CONCLUSIONS}

If Mach's principle is applicable to these data, then the marked consistency of the effects for two quite different classes of phenomena that share the same basic units that compose the universe would be expected. The essence of his principle is that local physical laws, such as those that apply to proton and electron movements involved with photon emissions and subtle shifts in $\mathrm{pH}$ of aqueous volumes are determined by and hence should reflect the structure of the universe. If the latter can be described in general by an expansion parameter, such as Hubble's constant, then there should be convergence between the solutions. The interface could involve the quantitative equivalence between gravity and electromagnetic fields correlated with light [11] as well as the possibility they are both 
affected by differences in dark matter pressures [12]. More advanced computer technologies that permit the full use of numerical intervals rather than integer values could test the precision of this method.

\section{References}

[1] E. Mach, The Science of Mechanics, The Open Court Publishing Co, Chicago, 1919.

[2] M. A. Persinger, S. A. Koren, International Journal of Neuroscience 117 (2007) 157-175.

[3] L. J. Martin, S. A. Koren, M. A. Persinger, Pharmacology, Biochemistry and Behavior 78 (2004) 217-227.

[4] N. J. Murugan, L. M. Karbowski, R. M. Lafrenie, M. A. Persinger, PLOSone, 2013 (online)

[5] L. S. St-Pierre, M. A. Persinger, International Journal of Neuroscience 116 (2006) 1-18.

[6] C. Buckner, Effects of electromagnetic fields on biological processes are spatial and temporal-dependent, Ph.D. Dissertation, Laurentian University, Sudbury, 2011.

[7] B. T. Dotta, M. A. Persinger, Journal of Biophysical Chemistry 3 (2010) 72-80.

[8] S. A. Koren, B. T Dotta, M. A. Persinger, The Open Astronomy Journal, in submission

[9] M. A. Persinger, K. S. Saroka, Journal of Electromagnetic Analysis and Applications 5 (2013) 151-156.

[10] T. E. Decoursey, Physiology Reviews 83 (2003) 475-579.

[11] M. A. Persinger, The Open Astronomy Journal 5 (2012) 41-43.

[12] T. Borowski, International Letters of Chemistry, Physics and Astronomy 11 (2013) 44-53. 